\title{
Changes of selected biochemical indicators and electrocardiography indicators in nutritional muscular dystrophy in calves
}

\author{
Katarzyna Żarczyńska ${ }^{1}$, Przemyslaw Sobiech ${ }^{1}$, Andrzej Pomianowski ${ }^{1}$, Agata Waclawska- \\ Matyjasik $^{1}$, Artur Stopyra ${ }^{1}$, Josef Illek ${ }^{2}$ \\ ${ }^{1}$ Department of Internal Diseases, Faculty of Veterinary Medicine, University of Warmia-Masury, \\ Olsztyn, Poland \\ ${ }^{2}$ Clinical Laboratory for Large Animals, Faculty of Veterinary Medicine, University of Veterinary \\ and Pharmaceutical Sciences, Brno, Czech Republic \\ Received September 7, 2011 \\ Accepted April 11, 2012
}

\begin{abstract}
The aim of this study was to estimate changes in selected biochemical indicators during nutritional muscular dystrophy of calves and to use electrocardiography technique in early diagnosis of this disease. The study was performed on 24 Holstein-Friesian calves of both sexes, divided into two groups. The first one was experimental group of 12 calves with symptoms of nutritional muscular dystrophy and the second one was control group of 12 clinically healthy calves administered a single i.m. injection of vitamin $\mathrm{E}+$ selenium preparation on the second day after birth. Blood samples were collected from all animals 3 $\times$ at 7-day intervals, starting on day 5 . Blood serum was subjected to biochemical analyses to determine Se and vitamin E concentrations, aspartate aminotransferase, creatine kinase and lactate dehydrogenase activity levels. The activity of glutathione peroxidase was determined in whole blood samples. Electrocardiographic assessment was performed on day 19 in all calves. In the group of calves with symptoms of nutritional muscular dystrophy, serum Se and vitamin E concentrations were significantly $(P \leq 0.01)$ lower than in control, the same was true for the activity of glutathione peroxidase $(P \leq 0.01)$. The changes were accompanied by an increase $(P \leq 0.01)$ in the activity of aspartate aminotransferase, creatine kinase and lactate dehydrogenase. The electrocardiograms of experimental animals revealed elevated heart rate, accelerated sinus rhythm, increased P wave amplitude, shorter PR, QT and ST interval, narrower QRS complex, shorter $\mathrm{T}$ wave duration and insignificantly increased $\mathrm{T}$ wave amplitude; these results were indicative of arrhythmia which is observed at early stages of cardiomyopathy. This study is the first one in which electrocardiography technique was used to diagnose nutritional muscular dystrophy in calves.
\end{abstract}

Cattle, selenium deficiency, biochemistry, arrhythmia

Selenium is a bio-element that plays a very important role in higher organisms. Selenium is nutritionally related to vitamin E and sulphur amino acids. Selenium and vitamin $\mathrm{E}$ deficiencies may lead to nutritional muscular dystrophy (NMD), also known as white muscle disease. The disorder affects lambs, foals, goatlings, calves and piglets, and is less frequently observed in mature animals. The disease involves hyaline degeneration of muscle cells in various skeletal muscles, including the diaphragm and the heart. In most calves, the disease appears at the age of 4-6 weeks. There are three main forms of nutritional muscular dystrophy: peracute, acute and subacute. The peracute form of the disease affects mainly the heart muscle of newborn calves. It is accompanied by arrhythmia, increased heart rate, weaker pulse, acute dyspnoea at rest, blue coloring of the mucosa and great breathing difficulty. In its acute form, the disease affects skeletal and intercostal muscles, the diaphragm and the heart. Calves have incorrect posture with widely spread limbs, hunched up back, neck extended forward with limited ability to move. In calves the subacute form of nutritional muscular dystrophy is most frequently encountered. The animals have

Address for correspondence:

Przemyslaw Sobiech

Department of Internal Diseases

Faculty of Veterinary Medicine, University of Warmia-Masury

Oczapowskiego 14, 10-957 Olsztyn, Poland

Phone: +48895233480

E-mail: psobiech@uwm.edu.pl

http://actavet.vfu.cz/ 
difficulty standing up, and degenerative changes are noted in the muscular coat of the tongue, throat and esophagus. In ruminants, subacute NMD is often diagnosed solely based on serum concentrations of AST, CK and LDH (Sobiech and Kuleta 1999).

Electrocardiography (ECG) is a highly useful diagnostic tool in cattle. The most common dysrhythmia in cattle is atrial fibrillation, the diagnosis of which can only be confirmed by ECG. Atrioventricular blocks, ventricular tachycardia, premature ventricular complexes and ventricular fibrillation have also been described in cows (Machida et al. 2005).

In ruminants, nutritional muscular dystrophy is also manifested by changes in the frequency and quality of heartbeat, and arrhythmia is often diagnosed. There is scarcity of data on ECG changes in ruminants caused by Se and vitamin E deficiencies. The above has been investigated only by Kojouri et al. (2009) who observed arrhythmia in lambs with white muscle disease.

The aim of this study was to determine changes in selected biochemical markers and ECG indicators in the clinical form of nutritional muscular dystrophy in calves.

\section{Materials and Methods}

The study was performed on 24 Holstein-Friesian calves of both sexes, at the age of two days of life and weighing about $40-45 \mathrm{~kg}$ at the beginning of experiment, divided into two groups: an experimental group of 12 calves with symptoms of nutritional muscular dystrophy and a control group of 12 clinically healthy calves administered a single, preventive i.m. injection of vitamin E + Se preparation (Eurovet Animal Health BV, Netherlands) comprising $50 \mathrm{mg} / \mathrm{ml}$ tocopherol acetate and $0.5 \mathrm{mg} / \mathrm{ml}$ sodium selenite at a dose of $2 \mathrm{ml} / 10 \mathrm{~kg}$ body weight on the second day after birth. The animals from both groups were subjected to clinical examinations, and blood samples were collected from the jugular vein $3 \times$ at 7 -day intervals, starting on day 5 (i.e. on day 5,12 and 19). Electrocardiographic assessment was performed on day 19 in all calves.

Blood serum samples were subjected to biochemical analyses to determine selenium and vitamin $\mathrm{E}$ concentrations, activity levels of aspartate aminotransferase (AST), creatine kinase (CK) and lactate dehydrogenase (LDH). Serum selenium concentrations were determined by flame atomic absorption spectrophotometry following hydride generation using the Unicam 939 Solar atomic spectrophotometer equipped with an Optimus data station, deuterium lamp background correction and a Unicam VP 90 hydride generation system. Vitamin E contents were determined by high-performance liquid chromatography (HPLC) with the use of the HP-1050 chromatograph and commercial Clin-Rep analytical kits (Recipal Chemical, Germany). Activity of AST was measured by the kinetic method using NADH and Tris buffer (Alpha Diagnostics, Poland), CK activity was measured by the kinetic method using NADH (Alpha Diagnostics, Poland), and LDH activity was measured by the kinetic method using $\mathrm{NAD}^{+}$and Tris buffer (Analco, Poland). The activity of glutathione peroxidase (GSH-Px) was determined in whole blood samples by the kinetic method with the use of cumene hydroperoxide and phosphate buffer (Ransel, United Kingdom). Biochemical analyses (except for Se estimation) were performed in the Laboratory of the Veterinary Faculty in Olsztyn, Poland. Selenium estimation was performed in the Laboratory of the Faculty of Veterinary Medicine, UVPS in Brno, Czech Republic.

Electrocardiography examinations were performed on standing animals in the shed, using the BTL-08 MT electrocardiograph. To insulate calves from the ground, rubber mats with a thickness of $2 \mathrm{~cm}$ were placed under the calves. Electrodes were attached to their skin folds in the following sequence: the positive electrode on the left side of the chest, in the fifth intercostal space at the level of the elbow; the negative electrode on the right side of the chest, at $2 / 3$ of the distance between the ramus of the mandible and the thoracic inlet and ground electrode on the right side of the medial plica. The output was recorded at paper speed of $25 \mathrm{~mm} / \mathrm{s}$ with $10 \mathrm{~mm} / \mathrm{mV}$ calibration.

The results of biochemical analyses and ECG were processed statistically by determining arithmetic means, standard deviations (SD) and significance of differences between measurements and groups at a confidence level of $P \leq 0.01$ using the Newman-Keulus test. Statistical analyses were performed with the use of Statistica software.

\section{Results}

Disease symptoms were not observed in the experimental group of calves (without administration of Se and vitamin E supplements) in the first days of their life. Signs of nutritional muscular dystrophy were reported in all experimental animals between days 10 and 17 . The initial symptoms were manifested by apathy, lethargic behaviour, decreased 
Table 1. Serum selenium and vitamin E concentrations and blood activity of glutathione peroxidase in experimental and control groups of calves $(\overline{\mathrm{x}} \pm \mathrm{SD})$

\begin{tabular}{lcccccc}
\hline \multirow{2}{*}{ Day of blood sampling } & \multicolumn{2}{c}{ Selenium $(\mu \mathrm{g} / \mathrm{l})$} & \multicolumn{2}{c}{ Vitamin $\mathrm{E}(\mu \mathrm{g} / \mathrm{ml})$} & \multicolumn{2}{c}{ GSH-Px $(\mathrm{U} / \mathrm{gHb})$} \\
\cline { 2 - 7 } & experimental calves & control group & experimental calves & control group & experimental calves & control group \\
\hline 5 & $19.92^{\mathrm{B}} \pm 2.96$ & $65.99^{\mathrm{A}} \pm 12.84$ & $2.89^{\mathrm{B}} \pm 0.78$ & $3.80 \pm 0.34$ & $32.24 \pm 6.94$ & $35.4^{\mathrm{A}} \pm 8.00$ \\
12 & $19.23^{\mathrm{B}} \pm 2.78$ & $106.23 \pm 16.52$ & $2.26^{\mathrm{B}} \pm 0.42$ & $3.39 \pm 0.30$ & $28.98^{\mathrm{B}} \pm 6.27$ & $67.8 \pm 23.52$ \\
19 & $18.80^{\mathrm{B}} \pm 2.82$ & $101.02 \pm 15.38$ & $2.03^{\mathrm{B}} \pm 0.28$ & $2.93 \pm 0.17$ & $22.23^{\mathrm{BA}} \pm 5.92$ & $144.0 \pm 36.40$ \\
\hline
\end{tabular}

A - significant difference between blood sampling $(P \leq 0.01)$, ${ }^{\mathrm{B}}$ - significant difference between groups $(P \leq 0.01), \overline{\mathrm{x}}$ - mean value, $\mathrm{SD}$ - standard deviation, GSH-Px - glutathione peroxidase

Table 2. Serum activity of aspartate aminotransferase, creatine kinase and lactate dehydrogenase in examined calves $(\overline{\mathrm{x}} \pm \mathrm{SD})$

\begin{tabular}{|c|c|c|c|c|c|c|}
\hline \multirow[t]{2}{*}{ Day of blood sampling } & \multicolumn{2}{|c|}{$\begin{array}{c}\text { AST } \\
(\mu \mathrm{kat} / \mathrm{l})\end{array}$} & \multicolumn{2}{|c|}{$\begin{array}{c}\text { CK } \\
(\mu \mathrm{kat} / \mathrm{l})\end{array}$} & \multicolumn{2}{|c|}{$\begin{array}{c}\mathrm{LDH} \\
(\mathrm{U} / \mathrm{l})\end{array}$} \\
\hline & experimental group & control group & experimental group & control group & experimental group & control group \\
\hline 5 & $0.79^{\mathrm{B}} \pm 0.06$ & $1.18 \pm 0.23$ & $3.50 \pm 0.23$ & $3.22 \pm 1.02$ & $3598.2 \pm 275.32$ & $3214.8 \pm 234.56$ \\
\hline 12 & $0.85 \pm 0.21$ & $0.93 \pm 0.26$ & $5.82^{\mathrm{BA}} \pm 0.72$ & $3.38 \pm 1.01$ & $4525.0^{\mathrm{BA}} \pm 429.01$ & $3089.7 \pm 251.36$ \\
\hline 19 & $1,57^{\mathrm{BA}} \pm 0.27$ & $0.91 \pm 0.29$ & $7.43^{\mathrm{BA}} \pm 0.46$ & $3.47 \pm 0.91$ & $4696.2^{\mathrm{BA}} \pm 335.37$ & $2876.8 \pm 209.44$ \\
\hline
\end{tabular}

A - significant difference between blood sampling $(P \leq 0.01),{ }^{\mathrm{B}}$ - significant difference between groups $(P \leq 0.01)$, $\overline{\mathrm{x}}$ - mean value, SD - standard deviation, AST - aspartate aminotransferase, CK - creatine kinase, LDH - lactate dehydrogenase

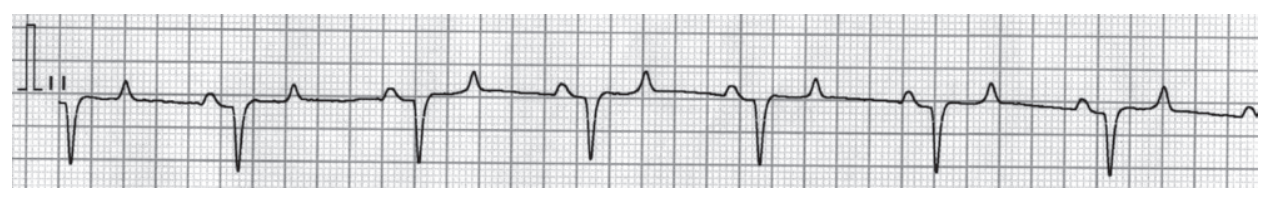

Fig. 1. Electrocardiography results of a healthy calf (paper speed $25 \mathrm{~mm} / \mathrm{s}$ with $10 \mathrm{~mm} / \mathrm{mV}$ calibration)

appetite and reluctance to move. They were followed by tremor in hind limbs, incorrect posture with widely spread limbs, hunched up spine, stilted gait and, in some animals, elevated respiratory rate and cough. Several animals remained recumbent and their body temperature exceeded $40{ }^{\circ} \mathrm{C}$.

In the experimental group, selenium concentration decreased from $19.92 \mu \mathrm{g} / 1$ on the $5^{\text {th }}$ day of experiment to $18.8 \mu \mathrm{g} / \mathrm{l}$ on the $19^{\text {th }}$, and it was significantly lower compared to the blood serum of the control group of calves throughout the experiment. In control animals, serum Se concentrations were marked by a significant increase to $106.23 \mu \mathrm{g} / \mathrm{l}$ at the second sampling and remained stable until the end of the experiment (Table 1). A moderate drop in serum vitamin E concentrations was observed in affected animals, from $2.89 \mu \mathrm{g} / \mathrm{ml}$ to 2.03 $\mu \mathrm{g} / \mathrm{ml}$ in the last evaluation. In the control group, vitamin E contents were significantly higher compared to diseased calves throughout the experiment, and they were marked by a moderate drop from $3.8 \mu \mathrm{g} / \mathrm{ml}$ to $2.93 \mu \mathrm{g} / \mathrm{ml}$ (Table 1).

Glutathione peroxidase activity in the blood of experimental animals showed a significant decrease from $32.24 \mathrm{U} / \mathrm{gHb}$ in the first blood sampling to $22.23 \mathrm{U} / \mathrm{gHb}$ in the 
third sampling. In the control group, the lowest level of GSH-Px activity observed in the first sampling $(35.41 \mathrm{U} / \mathrm{gHb})$ was followed by a significant increase to $144.03 \mathrm{U} / \mathrm{gHb}$ in the third sampling. On days 12 and 19, GSH-Px activity was significantly higher in healthy animals compared to the experimental group of animals (Table 1). Activity of AST in experimental animals was characterized by a significant increase, reaching $1.57 \mu \mathrm{kat} / 1$ in the last sampling, and a similar trend was reported with regard to the activity of CK (7.43 $\mu \mathrm{kat} / 1$ at the end of the experiment) and LDH (4496.2 U/1 on day 19) (Table 2). In the control group, AST, CK and LDH activity levels remained fairly constant throughout the experiment to reach $0.91 \mu \mathrm{kat} / 1,3.47 \mu \mathrm{kat} / 1$ and $2876.8 \mathrm{U} / 1$, respectively, in the last test. At the end of the experiment, enzyme activity levels were significantly higher in the serum of experimental animals than in control (Table 2).

The electrocardiograms of healthy animals were normal(Fig. 1). The electrocardiograms of experimental animals revealed elevated heart rate, normal and accelerated sinus rhythm, increased P wave amplitude, shorter PR interval, shorter QT interval, shorter ST interval, narrower QRS complex, shorter $\mathrm{T}$ wave duration and insignificantly increased $\mathrm{T}$ wave amplitude (Table 3, Figs 2, 3)

Table 3. Changes of selected electrocardiography indicators in calves with nutritional muscular disease and control calves $(\overline{\mathrm{x}} \pm \mathrm{SD})$

\begin{tabular}{|c|c|c|c|c|c|c|c|c|c|}
\hline & \multicolumn{9}{|c|}{ Indicators } \\
\hline & $\begin{array}{l}\text { Heart rate } \\
\text { (min) }\end{array}$ & $\mathrm{P}(\mathrm{s})$ & $\begin{array}{c}\text { PR } \\
\text { interval (s) }\end{array}$ & $\begin{array}{c}\text { PP } \\
\text { interval (s) }\end{array}$ & $\begin{array}{c}\text { RR } \\
\text { interval (s) }\end{array}$ & $\begin{array}{c}\text { QT } \\
\text { interval (s) }\end{array}$ & $\begin{array}{c}\text { ST } \\
\text { segment (s) }\end{array}$ & $\begin{array}{c}\text { QRS } \\
\text { complex (s) }\end{array}$ & $\mathrm{T}(\mathrm{s})$ \\
\hline $\begin{array}{l}\text { Experimental } \\
\text { group }\end{array}$ & $140 \pm 16$ & $0.048 \pm 0.012$ & $0.092 \pm 0.02$ & $0.39 \pm 0.05$ & $0.44 \pm 0.4$ & $0.213 \pm 0.03$ & $0.08 \pm 0.02$ & $0.073 \pm 0.007$ & $0.079 \pm 0.005$ \\
\hline Control group & $120 \pm 10$ & $0.042 \pm 0.015$ & $0,11 \pm 0.01$ & $0.42 \pm 0.03$ & $0.46 \pm 0.2$ & $0.251 \pm 0.05$ & $0.09 \pm 0.03$ & $0.082 \pm 0.005$ & $0.082 \pm 0.003$ \\
\hline
\end{tabular}

$\overline{\mathrm{X}}$ - mean value, $\mathrm{SD}$ - standard deviation

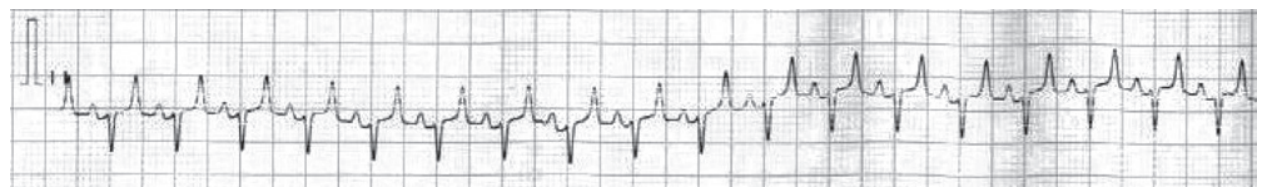

Fig. 2. Electrocardiography results of a calf with nutritional muscular dystrophy - sinus tachycardia (paper speed $25 \mathrm{~mm} / \mathrm{s}$ with $10 \mathrm{~mm} / \mathrm{mV}$ calibration)

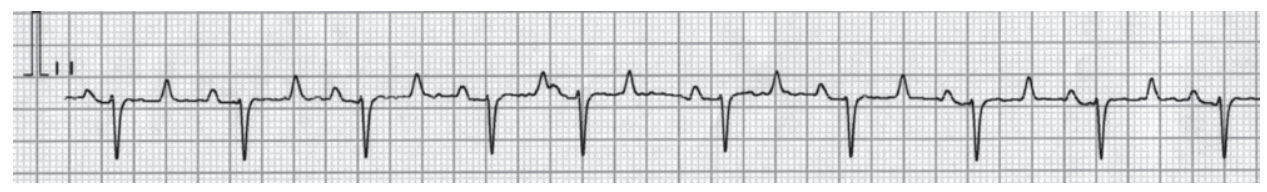

Fig. 3. Electrocardiography results of a calf with nutritional muscular dystrophy - sinus arrhythmia and tachycardia ( paper speed $25 \mathrm{~mm} / \mathrm{s}$ with $10 \mathrm{~mm} / \mathrm{mV}$ calibration) 


\section{Discussion}

In the experimental group, Se concentrations decreased with age and the progression of muscular dystrophy. Based on the referenced data (Pavlata et al. 2005), Se concentrations in the studied experimental calves should be regarded as very low and indicative of acute hyposelenaemia. The highest Se concentrations in the control group were noted rather late, at the second blood sampling, i.e. two weeks after supplementation. These results are similar to data obtained by Weiss et al. in calves (1983), but are in contrast to data obtained by Slavik et al. in cows (2008). Results published by Blodget and Bevill (1987) proved that serum Se concentration after parenteral administration can be dependent on the content of its inhibitors (arsenic, sulfur or phosphorus). Contents of these elements were not obtained in our study.

In calves affected by NMD, $\alpha$-tocopherol concentrations decreased with age and disease progression. Physiological serum concentrations of vitamin $E$ in young ruminants are determined by its content in the colostrum and milk because vitamin $\mathrm{E}$ is practically not transferred across the placenta (Beytut et al. 2002). In our study, vitamin E concentrations determined in the serum of diseased calves at the end of the experiment were significantly below the physiological norm, pointing to vitamin E deficiency. In the serum of healthy animals, vitamin E concentrations decreased steadily throughout the experiment. Vitamin E concentrations remained elevated over a short period of time; therefore, additional supplementation is required, for example, at weekly intervals.

Glutathione peroxidase activity (GSH-Px) in blood of experimental calves decreased with the progression of muscular dystrophy symptoms. In ruminants, NMD symptoms can be expected to appear when GSH-Px activity levels drop below $25 \mathrm{U} / \mathrm{gHb}$ (Bickhardt et al. 1999). In our study, GSH-Px activity levels in experimental calves dropped below $23 \mathrm{U} / \mathrm{gHb}$ at the last sampling, indicating that the discussed enzyme plays a significant role in the pathogenesis of NMD. In healthy animals GSH-Px activity increased significantly 2.5 weeks after Se injection. The results are similar to our previous investigations performed on lambs (Sobiech and Kuleta 1999). Activity of this enzyme is connected with the lifespan of erythrocytes, but not all factors influencing GSH-Px activity in blood are known e.g. the role of selenoprotein P or selenoprotein W (Whanger 2000). It should also be noted that GSH-Px activity continued to increase in the third test despite a drop in Se concentrations. Similar data were obtained by Beytut et al. (2002) who demonstrated that GSH-Px activity is a more reliable indicator of Se concentrations in its deficiency than in a state of adequate Se supply.

The average level of AST activity in the experimental group at the last examination was determined at $1.57 \mu \mathrm{kat} / \mathrm{l}$ which exceeds the physiological norm for the species (0.92 $\mu \mathrm{kat} / \mathrm{l}$, Dubreuil and Lapierre 1997), but is lower than the values reported in other studies of NMD (Abutarbush and Radostits 2003). The highest increase in enzyme activity was observed in horses with NMD where AST levels reached several thousand U/1 (Ludvikova et al. 2007). The levels of AST activity noted in our study were much lower, indicating that this enzyme exhibits lower specificity for muscular tissue in calves, and that it is a less effective bioindicator of nutritional muscular dystrophy.

Creatine kinase, considered to be the most sensitive bioindicator of degenerative muscle disease, showed the highest affinity for muscular tissue (Kohli et al. 2005). A permanent rise in $\mathrm{CK}$ activity is indicative of disease, and it is correlated with the degree of pathological changes. In our study, an increase in CK activity was noted in 
the serum of calves diagnosed with NMD at the last two samplings, and the steepest increase was reported at the end of the experiment. The above points to progressive muscle degeneration but the noted values are significantly below those reported by other authors investigating nutritional muscular dystrophy in ruminants (Bickhardt et al. 1999). The above difference can be attributed to the degree of disease progression as well as significant variations in CK activity levels between individuals.

The activity of lactate dehydrogenase in the blood serum of affected calves increased throughout the experiment, and the results of the last two tests were significantly higher than in control. Lactate dehydrogenase is not specific for muscular tissue, and its activity in the blood serum of ruminants may also increase due to liver disease, skeletal dysfunctions and digestive disorders (Sobiech et al. 2004; Sobiech and Kuleta 2006). In our study, an increase in LDH activity in the serum of experimental calves was indicative of dystrophic changes in muscular tissue, and it was correlated with the observed clinical symptoms.

The ECG results of calves diagnosed with NMD are indicative of arrhythmia which is observed at early stages of cardiomyopathy. Electrocardiography changes in our study corresponded with the results of a similar experiment involving lambs (Kojouri et al. 2009) but cardiac dysfunctions were less pronounced, which could be attributed to earlier stages of the disease. Our results indicate that the acute form of nutritional muscular disease in calves is accompanied by degenerative changes in the heart muscle, making electrocardiography the diagnostic method of choice.

The results of our study indicate that nutritional muscular dystrophy in calves is characterized by a drop in serum Se and vitamin E concentrations, decreased GSHPx activity and increased activity of AST, CK and LDH. The disease is accompanied by cardiac dysrhythmia of various intensity; therefore, ECG examinations should be included in the protocol for routine diagnosis of nutritional muscular dystrophy in calves.

\section{References}

Abutarbush SM, Radostits OM 2003: Congenital nutritional muscular dystrophy in a beef calf. Can Vet J 44: 738-739

Beytut E, Karatas F, Beytut E 2002: Lambs with white muscle disease and selenium content of soil and meadow hay in the region of Kars. Turkey Vet J 163: 214-217

Bickhardt K, Ganter M, Sallman P, Fuhrmann H 1999: Investigations on manifestations of vitamin E and selenium deficiency in sheep and goats. Dtsch Tierarztl Wochenschr 106: 242-247

Blodgett DJ, Bevill RF 1987: Pharmacokinetics of selenium administered parenterally at toxic doses in sheep. Am J Vet Sci 48: 530-534

Dubreuil P, Lapierre H 1997: Biochemistry reference values for Quebec lactating dairy cows, nursing sows, growing pigs and calves. Can J Vet Res 61: 235-239

Kohli R, Harris DC, Whitington PF 2005: Relative elevation of serum alanine and aspartate aminotransferase in muscular dystrophy. J Pediatr Gastroenterol Nutr 41: 121-124

Kojouri GA, Rezakhani A, Ahmadi H 2009: Arrhythmias in advance stiff lamb disease. Small Rumin Res 84: 65-69

Ludvikova E, Jahn P, Lukas Z 2007: Nutritional myodegeneration as a cause of dysphagia in adult horses: three case reports. Vet Med-Czech 52: 267-272

Machida N, Katsuda S, Koyabashi Y, Mitsumori K 2005: A histological study of the cardiac conduction system in a heifer with complete atrioventricular block. J Comp Med 133: 68-72

Pavlata L, Podhorsky A, Pechova A, Dvorak R 2005: Incidence of hypovitaminosis E in calves and therapeutic remedy by selenium-vitamin supplementation. Acta Vet Brno 74: 209-216

Slavik P, Illek J, Rajmon R, Zeleny T, Jilek F 2008: Selenium dynamics in the blood of beef cows and calves fed diets supplemented with organic and inorganic selenium sources and the effect on their reproduction. Acta Vet Brno 77: 11-15

Sobiech P, Kuleta Z 1999: Levels of selected biochemical indicators of serum and blood during subclinical form of nutritional muscular dystrophy in lambs. Polish J Vet Sci 2: 37-41

Sobiech P, Kuleta Z 2006: Activity of LDH isoenzymes in diarrhoeic calves. Bull Vet Inst Pulawy 50: 401-404 
Sobiech P, Kuleta Z, Stopyra A 2004. Serum LDH isoenzyme activity in dairy and beef cattle. Indian Vet J 81: 755-756

Weiss WP, Colenbrander VF, Cunningham MD, Callahan CJ 1983: Selenium/Vitamin E: Role in disease prevention and weight gain of neonatal calves. J Dairy Sci 66: 1101-1107

Whanger PD 2000: Selenoprotein W: a review. Cell Mol Life Sci 57: 1846-1852 